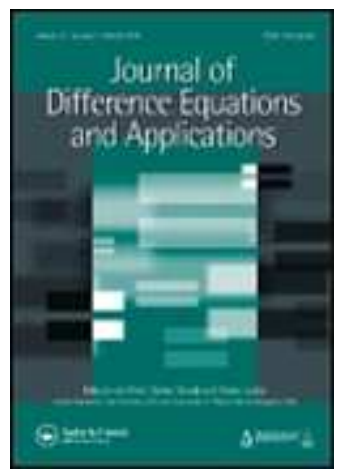

\title{
A Lyness equation for graphs
}

\begin{tabular}{|r|l|}
\hline Journal: & Journal of Difference Equations and Applications \\
\hline Manuscript ID: & Draft \\
\hline Manuscript Type: & Original Article \\
\hline Date Submitted by the & n/a \\
Author: & \\
\hline Complete List of Authors: & Northshield, Sam; SUNY, Mathematics \\
\hline Keywords: & difference equation, graph, periodicity, invariant \\
\hline
\end{tabular}

\section{SCHOLARONE ${ }^{m}$ Manuscripts}




\title{
A Lyness equation for graphs
}

\author{
Sam Northshield
}

is of degree 2 in the sense that any two adjacent values, say $x_{0}$ and $x_{1}$ determine every $x_{n}, n \in \mathbb{Z}$. That is, the space of solutions is two-dimensional.

A remarkable property is the fact that every solution of (1) is, when $k=1$, periodic of period 5: for all $n$ :

$$
x_{n+5}=x_{n} .
$$

Similarly, when $k=0$, every solution is of period 6 . It can be argued that (1) makes sense when $k=\infty$ (making (1) into $x_{n+1}=1 / x_{n-1}$ ) which is periodic with period 4 (see Zeeman [6]).

These turn out to be the only values of $k$ for which all solutions are periodic. However, for every $k$, solutions of (1) exhibit a type of pseudo-periodicity: the 
set of points $\left\{\left(x_{n}, x_{n+1}\right): n \in \mathbb{Z}\right\}$ lie on a simple closed curve. To see this, note that for any $k$, the function

$$
F(x, y):=\frac{(x+y+k)(x+1)(y+1)}{x y}
$$

satisfies, for any solution of (1), $F\left(x_{n-1}, x_{n}\right)=F\left(x_{n}, x_{n+1}\right)$ (see Section 4). Hence, for some $C$ depending on $x_{0}$ and $x_{1}$, every point $\left(x_{n}, x_{n+1}\right)$ satisifies

$$
x^{2} y+x y^{2}+x^{2}+y^{2}+C x y+(x+y)(k+1)+k=0 .
$$

We call a function $F$ an invariant. The curve defined by (2) is a cubic curve and thus has a group law and this fact has been exploited by Bastien and Rogalski [2] to show that there are periodic solutions of all but finitely many periods. That is, for all but finitely many $n$, there exist $k, x_{0}, x_{1}$ such that the resulting solution of (1) has period $n$.

A simple graph is a countable set (of vertices) with a binary relation $\sim$ such that $x \neq x$ for all $x$. If $x \sim y$ we say that $x$ and $y$ are adjacent or that there is an edge between $x$ and $y$ or that $x$ is a neighbor of $y$. We denote the set of neighbors of $y$ by $N_{y}$. The set of integers $\mathbb{Z}$ with $m \sim n$ iff $|m-n|=1$ is a simple graph. If one thinks of each vertex as a variable then the Lyness equation (1) can be written as

$$
x z=y+k \text { if } N_{y}=\{x, z\} .
$$

We may attempt to generalize (3) to other graphs. For example, (3) makes sense for any 2-regular graph. It turns out that every 2-regular simple graph is either (isomorphic to) the 2-regular tree $T_{2}$ or a cycle $C_{n}$ ( $n$ being the length of the cycle or, equivalently, the number of vertices in it). The work of Bastien and Rogalski says that equation (3) has a solution for all but finitely many $n$ (with $k$ depending on $n$ ). The older work of Lyness shows that when $k=1$ the solutions of (3) on $T_{2}$ are in one-to-one correspondence to solutions on $C_{5}$. Similarly, when $k=0$, the solutions of (3) of $T_{2}$ are in one-to-one correspondence with solutions of (3) on $C_{6}$.

The plan for the remainder of this paper is to attempt to generalize these facts. We first discuss more general graphs in section 2. In section 3, we define the Lyness equation on 3-regular graphs and find some periodic solutions. In section 4 , we find invariants and, in section 5 , discuss some open problems and directions of future research.

\section{Graphs and coverings}

We shall consider a graph to be a countable set (of vertices) with a binary relation $\sim: x \sim y$ if there is an edge connecting $x$ and $y$. We say that $x$ and $y$ are adjacent if $x \sim y$. We shall assume that a graph is simple: $x \nsim x$ for all $x$ and there is at most one edge between any two vertices. 
For a vertex $x$, we define its neighborhood set:

$$
N_{x}=\{y: y \sim x\} .
$$

A graph is $n$-regular if every vertex has exactly $n$ neighbors.

A tree is an acyclic connected graph [1]. That is, for any distinct vertices $x, y$ there is a unique sequence $x_{0}, x_{1}, \ldots, x_{n}$ of distinct vertices such that $x_{0}=x$, $x_{n}=y$, and $x_{i-1} \sim x_{i}(i=1, \ldots, n)$. There is, for every $n$, a unique (up to graph isomorphism) $n$-regular tree $T_{n}$. The set $\mathbb{Z}$ with adjacency relation $m \sim n$ iff $|m-n|=1$ is a realization of $T_{2}$.

Given two graphs $G$ and $H$, we say that $G$ covers $H$ if there is a function from $G$ to $H$ (we write $\theta: G \rightarrow H$ ) which preserves adjacency and vertex degree. Specifically, this means that if $u \sim v$ in $G$ then $\theta(u) \sim \theta(v)$ in $H$ and, if $u$ has $n$ neighbors in $G$, then $\theta(u)$ has $n$ neighbors in $H$. Topologically, this is just saying that $\theta$ is a restriction (to the vertex set) of a local homeomorphism. The tree $T_{2}$ covers every cycle (we say that $T_{2}$ is the universal covering tree of a cycle). Similarly, every $n$-regular graph is covered by $T_{n}$.

Suppose $\theta: G \rightarrow H$ is a graph covering. The lift of a function $f: H \rightarrow \mathbb{R}$ is the function $f \circ \theta: G \rightarrow \mathbb{R}$. A function $f: G \rightarrow \mathbb{R}$ is said to be periodic if it is the lift of some function $f: H \rightarrow \mathbb{R}$ where $H$ is covered (non-trivially) by $G$. For example, a real function $f$ on $T_{2}=\mathbb{Z}$ is periodic if and only if it is a lift of a function on some cycle $C_{n}$ and thus $f(i+n)=f(i)$ for all $i$.

\section{The Lyness equation on graphs}

The Lyness equation (3) may be rewritten as an equation on $T_{2}$ :

$$
x z=y+k,\left[N_{y}=\{x, z\}\right] .
$$

This equation actually makes sense on any finite 2-regular graph (cycle) as well.

This leads to a way to define Lyness equations for $n$-regular graphs:

$$
S\left(N_{x}\right)=x+k
$$

where $S(N)$ is a fixed symmetric function on (unordered) $n$-tuples. For example, $x y+x z+y z=w+k$ or $x y z=w+k,\left[N_{w}=\{x, y, z\}\right]$. The finite dimensionality of the solution space in the 2-regular case is desirable but totally absent in the 3 -regular case. It is thus desirable to make the space of solutions on $T_{3}$ finite dimensional; we shall do this by including an auxiliary equation. Hence we shall consider equations of the form

$$
\left\{\begin{array}{l}
S\left(N_{x}\right)=x+k \\
L\left(N_{x}\right)=f(x) .
\end{array}\right.
$$

where $L(\{x, y, z\})=x+y+z, f$ is a function, and either $S(\{x, y, z\})=x y z$ or $S(\{x, y, z\})=x y+x z+y z$. 
Consider first, for a given function $f$,

$$
\left\{\begin{array}{l}
x y z=w+k \\
x+y+z=f(w)
\end{array} \quad\left[N_{w}=\{x, y, z\}\right] .\right.
$$

Then $x+y=f(w)-z$ and $x y=(w+k) / z$. Solving for $x, y$ by finding the roots of $(T-x)(T-y)=T^{2}-(x+y) T+x y=T^{2}-(f(w)-z) T+(w+k) / z=0$,

$$
x, y=\frac{1}{2}\left[f(w)-z \pm \sqrt{(f(w)-z)^{2}-4(w+k) / z}\right] .
$$

Hence, knowing $w$ and $z$ determines, up to graph automorphism, the value at the other two neighbors of $w$ and thus, inductively, the solution at all locations. That is, any two adjacent values determine the entire solution (modulo graph automorphism).

We now consider some periodic solutions of (5). For example, let $S(x, y, z):=$ $x y z$ and, on the tetradhedron (the unique 3-regular graph with 4 vertices), consider $S\left(N_{w}\right)=w$. This equation has a solution which is 1 on (any) two vertices and -1 on the other two. Hence, the lift is periodic on $T_{3}$.

Let $S(x, y, z):=x y+x z+y z$ and, on the cube (the unique 3-regular bipartite graph with 8 vertices), consider $S\left(N_{w}\right)=w+\frac{7}{3}$. Assigning to adjacent vertices the distinct values $-1, \frac{2}{3}$ gives a solution on the cube and thus lifts to a periodic solution on $T_{3}$.

These two examples show two different types of periodicity. A natural question is whether there are any $S$ and $k$ such that every solution of $S\left(N_{x}\right)=x+k$ is the lift of a solution on a particular fixed finite 3-regular graph (analogous to every solution of the Lyness equation with $k=1$ is the lift of a solution on the 5-cycle). This question is actually rather easy to answer as stated. For a fixed $S$ and $k$ in the 3 -regular case, it is possible to choose a solution such that every vertex is assigned a distinct real value and thus making it non-periodic. Similarly, since there is always extra degrees of freedom when constructing a solution, the existence of an invariant is impossible.

Proposition 3.1. The Lyness type equation

$$
\left\{\begin{array}{l}
x y z=w^{3} \\
x y+x z+y z=-w^{2}, \quad\left[N_{w}=\{x, y, z\}\right] \\
x+y+z=-w
\end{array}\right.
$$

has every solution satisfying tetrahedral symmetry.

Proof. By the hypothesis,

$$
(t-x)(t-y)(t-z)=(t-w)(t+w)^{2}
$$

and so, of the 3 neighbors of a vertex $w$, one takes the same value as $w$ and two take the negative of that value. 
Proposition 3.2. For a real valued function $f$, every real solution of

$$
\left\{\begin{array}{l}
x y+x z+y z=3 f(w)^{2}, \\
x+y+z=3 f(w)
\end{array} \quad\left[N_{w}=\{x, y, z\}\right],\right.
$$

has cubic symmetry.

Proof. It is enough to show that for any $w$, any solution is constant on $N_{w}$. Suppose $N_{w}=\{x, y, z\}$. Then

$$
x^{2}+y^{2}+z^{2}+2(x y+x z+y z)=(x+y+z)^{2}=9 f(w)^{2}=3(x y+x z+y z)
$$

and so $x^{2}+y^{2}+z^{2}-x y-x z-y z=0$. Solving for, say, $z$ yields

$$
z=\frac{x+y}{2} \pm i \frac{\sqrt{3}(x-y)}{2}
$$

and thus, since $x, y, z$ are real, $x=y$. The result follows.

\section{Invariants}

In this section, we review the Lyness equation on $T_{2}$ in more detail as well as properties of invariants associated to it. First, notice that the space of solutions of the Lyness equation is two-dimensional in the sense that knowing values at any two adjacent vertices determines the entire solution. Further, there exists an invariant $I(x, y)$ which is constant for any two adjacent vertices $x, y$ in a given solution. Hence every point $(x, y)$ associated to two such adjacent vertices lies on a level curve $I(x, y)=C$ which turns out to be a bounded cubic curve.

Let's recall two strategies for finding invariants in the 2-regular case. Assume $x, y, z$ are variables satisfying $x z=y+k$. We say a function is "type $F$ " if it a function of $x, y, z$ invariant under switching $x$ and $z$. We say a function is "type $G$ " if it is a function of $y, z$ invariant under switching $y, z$. If we can find two such functions satisfying

$$
F(x, y, z)=G(y, z)
$$

then $G(x, y)=G(y, z)$ and so $G$ is an invariant.

As an example, the function $(x+1)(z+1)$ is type $F$ and, rewriting by using $x z=y+k$, we find

$$
(x+1)(z+1)=\frac{(x z+z)(z+1)}{z}=\frac{(y+z+k)(z+1)}{z} .
$$

The right side is a function involving just $y$ and $z$ but is not symmetric. It is possible to multiply both sides by a function of $y$ to make the right side of type $G$ but keeping the left type $F$ :

$$
\frac{(x+1)(y+1)(z+1)}{y}=\frac{(y+z+k)(z+1)(y+1)}{x y} .
$$


Hence,

$$
I(x, y)=\frac{(x+y+k)(x+1)(y+1)}{x y}
$$

is an invariant for the Lyness equation (1),(3).

Another strategy is to find two functions $G_{1}, G_{2}$ of type $G$ and a function $h$ of a single variable such that

$$
G_{1}(x, y)=G_{2}(y, z) h(z) .
$$

If so, then switching $x$ and $z$ yields

$$
G_{1}(z, y)=G_{2}(y, x) h(x) .
$$

Finally, cross-multiplying yields

$$
G_{1}(x, y) G_{2}(x, y) h(y)=G_{1}(y, z) G_{2}(y, z) h(z)
$$

and therefore, multiplying both sides by $h(y)$ yields the invariant

$$
F(x, y):=G_{1}(x, y) G_{2}(x, y) h(x) h(y) .
$$

As an example of this technique, again let $x z=y+k$. Then

$$
(x+y+1) z=x z+y z+z=y+z+y z+k
$$

and so

$$
x+y+1=(y+z+y z+k) \cdot \frac{1}{z}
$$

which is of the form of (6). Hence,

$$
F(x, y):=\frac{(x+y+1)(x y+x+y+k)}{x y}
$$

is (another) invariant for the Lyness equation. Note that the difference between the two Lyness invariants is constant.

If two different invariants exist that do not differ by a constant, then the points $\left(x_{n}, x_{n+1}\right)$ must lie on the intersections of two level curves which possibly intersect only at finitely many points. This perhaps this leads to a new proof of the periodicity of solutions of (2) when $k=0$ or 1 .

As another example, consider the Somos-5 sequence (see [3]) defined by the recursion

$$
x_{n} x_{n+5}=x_{n+1} x_{n+4}+x_{n+2} x_{n+3} .
$$

Remarkably, for starting values $x_{i}=1$ for $i=1,2,3,4,5$, every $x_{i}$ is an integer. Zagier $[\mathrm{Z}]$ noted that for

$$
y_{n}:=\frac{x_{n} x_{n+3}}{x_{n+1} x_{n+2}},
$$

$y_{n-1} y_{n} y_{n+1}=y_{n}+1$. As above, we rewrite this equation as $x y z=y+1$. Then

$$
x y+1=(y+z+1) \cdot \frac{1}{z}
$$


and thus an invariant is

$$
I(x, y)=\frac{(x y+1)(x+y+1)}{x y} .
$$

That is, when $\left(x_{n}\right)$ is a Somos-5 sequence, then the following quantity is independent of $n$ :

$$
\frac{\left(x_{n} x_{n+4}+x_{n+2}^{2}\right)\left(x_{n+1} x_{n+2} x_{n+3}+x_{n} x_{n+3}^{2}+x_{n+4} x_{n+1}^{2}\right)}{x_{n} x_{n+1} x_{n+2} x_{n+3} x_{n+4}} .
$$

We next find invariants for the general case. One strategy is to seek two functions $F, G$ satisfying

$$
F(\{x, y, z\}, w)=G(\{x, w\}),\left[N_{w}=\{x, y, z\}\right] .
$$

That is, $F$ is invariant under permutations of $x, y, z$ and $G$ is symmetric. By the symmetry of $F$, we may switch $x$ and either $z$ or $y$. Since $T_{3}$ is connected, $G$ is an example of the desired invariant.

Proposition 4.1. The Lyness equation

$$
\left\{\begin{array}{l}
x y z=w+k \\
x+y+z=\frac{1}{w}
\end{array} \quad\left[N_{w}=\{x, y, z\}\right]\right.
$$

has $I(a, b):=\frac{a^{2}+b^{2}-a^{3} b-b^{3} a+k a+k b}{a b}$ constant for all pairs of adjacent values $a, b$.

Proof. We shall find $F$ and $G$ satisfying $F(\{x, y, z\}, w)=G(\{x, w\})$.

$$
\begin{aligned}
x y+x z+y z & =x(y+z)+y z \\
& =x\left(\frac{1}{w}-x\right)+\frac{w+k}{x} \\
& =\frac{x}{w}+\frac{w}{x}-x^{2}+\frac{k}{x}
\end{aligned}
$$

and therefore

$$
x y+x z+y z-w^{2}+\frac{k}{w}=\frac{x}{w}+\frac{w}{x}-x^{2}-w^{2}+\frac{k}{x}+\frac{k}{w}
$$

and the result follows.

Proposition 4.2. The Lyness equation

$$
\left\{\begin{array}{l}
x y+x z+y z=w+k \\
x+y+z=s w^{2}+t
\end{array} \quad\left[N_{w}=\{x, y, z\}\right]\right.
$$

has $I(a, b):=a^{3}+b^{3}-s a^{2} b^{2}-t\left(a^{2}+b^{2}\right)+a b+k(a+b)$ constant for all pairs of adjacent values $a, b$. 
Proof. Again, we shall find $F$ and $G$ satisfying $F(\{x, y, z\}, w)=G(\{x, w\})$.

$$
\begin{aligned}
x y z & =x(w+k-x(y+z)) \\
& =x\left(w+k-x\left(s w^{2}+t-x\right)\right) \\
& =x w+k x-s x^{2} w^{2}+t x^{2}+x^{3}
\end{aligned}
$$

and therefore

$x y+x z+y z-+k w-t w^{2}+w^{3}=x w+k x+k w-s x^{2} w^{2}-t x^{2}-t w^{2}+x^{3}+w^{3}$

and the result follows.

Zeeman noted [6] that the Lyness equation on $T_{2}$ is periodic with period 4 for every solution when $k=\infty$. This, of course, is somewhat informal. However, we see that if, in the previous example, we let $k$ grow without bound, then $I(a, b)=a+b$ is an invariant. That is, every solution of

$$
x y+x z+y z=w+\infty, \quad\left[N_{w}=\{x, y, z\}\right]
$$

is such that every solution is constant on every neighbor set $N_{w}$ and therefore the solution exhibits cubic symmetry.

A special case of Proposition 4.2 is the following.

Proposition 4.3. The Lyness equation

$$
\left\{\begin{array}{l}
x y+x z+y z=w+k \\
x+y+z=0
\end{array} \quad\left[N_{w}=\{x, y, z\}\right],\right.
$$

has $I(a, b):=a^{3}+b^{3}+k(a+b)+a b$ constant for all pairs of adjacent values $a, b$.

The following replaces both equations of (5) by variants.

Proposition 4.4. The Lyness equation

$$
\left\{\begin{array}{l}
x y+x z+y z=c_{1} / w \\
x y z=c_{2} / w^{2}
\end{array} \quad\left[N_{w}=\{x, y, z\}\right]\right.
$$

has $I(a, b):=a+b+\frac{c_{1}}{a b}+\frac{c_{2}}{a^{2} b^{2}}$ constant for all pairs of adjacent values $a, b$.

In general, knowing the value of the invariant for a specific solution along with the value at a single vertex determines the entire solution. For example, if $I=3$ in the case above and a single value $a$, we can solve

$$
a^{2} x^{3}+a^{2}(a-3) x^{2}+c_{1} a x+c_{2}=0
$$

to get the values at three adjacent vertices. 
In equation (1), the solution is always bounded and real (for real initial values $\left.x_{0}, x_{1}\right)$. This is because each point $\left(x_{n}, x_{n+1}\right)$ is restricted to a level curve of the invariant which is of the form $(x+y+k)(x+1)(y+1)=C x y$ which turns out to be a closed and bounded. In the more general case, this is no longer true. For example, Figure 1 shows the level curves for the invariant $I(x, y)=x^{3}+y^{3}+x y$ of the equation

$$
\left\{\begin{array}{l}
x y+x z+y z=w \\
x+y+z=0
\end{array} \quad\left[N_{w}=\{x, y, z\}\right]\right.
$$

Although it is true that every real $(x, y)$ for adjacent values in a solution of (7) lies on one of these level curves, it is not true that every solution is entirely real.

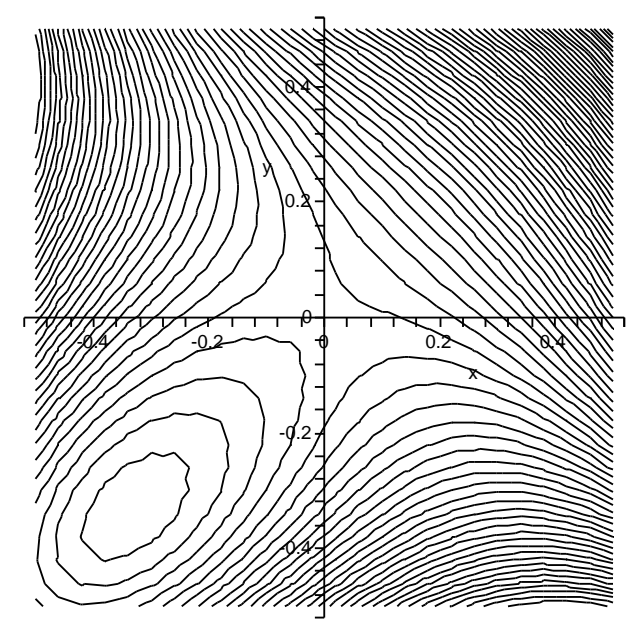

Figure 1: Level curves for $x^{3}+y^{3}+x y$

For example, consider (7) with two given values for $x$ and $w$. Then $(t-$ $y)(t-z)=t^{2}-(y+z) t+y z=t^{2}+x t+x^{2}+w$ and therefore

$$
y, z=\frac{-x \pm \sqrt{-3 x^{2}-4 w}}{2} .
$$

If the solution is purely real, then it is purely non-positive since, otherwise, some vertex $w$ is negative and two of its neighbors must then take on complex values. This is impossible however since $x<0$ implies $-x+\sqrt{-3 x^{2}-4 w}>0$. Therefore, the only real solution is identically 0 . 


\section{Open questions}

1) Are there any equations, as in Propositions 3.1 and 3.2, with dodecahedral symmetry?

2) For what finite graphs is there a non-trivial solution of (5)?

3) What conditions (if any) on (5) ensure that all real solutions are constant?

4) What equations (5) have an invariant?

\section{References}

[1] J. Bondy and U. Murty, Graph Theory with Applications, North-Holland, New York, 1976.

[2] G. Bastien and M. Rogalski, Global Behavior of the Solutions of Lyness' Difference Equation $u_{n+2} u_{n}=u_{n+1}+a$, J. Difference Equations and Appl., 10,no. 11, (2004), 977-1003.

[3] J. Propp, The Somos Sequence Site, http://faculty.uml.edu/jpropp/ somos.html.

[4] H. Wilf, generatingfunctionology, Academic Press, Boston, 1990.

[5] D. Zagier, Solution (Day 5, problem 1), St. Andrews Meeting, http:// www-groups.dcs.st-and.ac.uk/ john/Zagier/Solution5.1.html.

[6] C. Zeeman, Geometric Unfolding of a Difference Equation, http:// zakuski.utsa.edu/ gokhman/ecz/gu.html

Department of Mathematics, SUNY, Plattsburgh, NY 12901

northssw@plattsburgh.edu 